\title{
Mirada a la educación superior en Venezuela
}

\author{
Claudio Rama
}

Doctor en Ciencias de la Educación y en Derecho. Profesor, investigador y Consultor Universitario. Fue decano de la Facultad de Ciencias Empresariales de la Universidad de la Empresa (UDE), ex-Director del Instituto Internacional para la Educación Superior en América Latina y el Caribe (IESALC-UNESCO).

\section{Introducción}

En este número especial, dedicado al análisis de la educación superior en Venezuela, participan algunos de los más connotados especialistas del país, incluida la inestimable colaboración de Tulio Ramírez de la Universidad Central de Venezuela y, al tiempo, coordinador del Doctorado en Educación de la Universidad Católica Andrés Bello (UCAB). Los artículos aquí presentados nos permiten tener una visión amplia y rigurosa para caracterizar la situación de la educación superior en Venezuela. Sin duda, podrían faltar aristas como en toda selección dado el espacio limitado de la revista, no solo por la alta complejidad que tiene la educación superior, sino también por la existencia de diversas miradas en un contexto altamente polarizado.

Cada uno de nosotros tiene su propia visión y lectura. Temas como evaluación, regulación, acreditación e investigación son vistos tangencialmente. Igualmente la educación privada, tecnológica o de posgrado no son abordadas con análisis particulares. La internacionalización o la regionalización también podrían ser vistas más atentamente. En este sentido, estamos frente a un número que selecciona algunos temas y enfoques, como acontece en cualquier análisis, en tanto siempre se reduce la realidad que es más vasta.

Esta introducción la hacemos por la alta polarización que respecto a la educación superior, y específicamente a la realidad venezolana, existe entre algunos sectores. Muchas veces por escasa información y otras por criterios previos. Quienes, como yo, han vivido, conocido e investigado la realidad de la educación superior venezolana -como estudiante, profesor y representante estudiantil y de egresados en nuestra juventud y a lo largo de 10 años, y luego como director del Instituto Internacional de la UNESCO para la Educación Superior en América Latina y el Caribe (IESALC) con sede en Venezuela-, tenemos una visión más amplia, más allá de la subjetividad, que parte de los enfoques de las ciencias sociales, al menos en la selección de las informaciones y análisis. En los años 2000 impulsamos una de las más amplias investigaciones sobre la educación superior en Venezuela que 
se expresó en una colección de 13 libros que prácticamente sirvió de referencia en su momento y que se constituye en la línea de base sobre la cual se puede intentar comprender la compleja, contradictoria y, a la vez limitada en datos, dinámica de la universidad venezolana.

La visión histórica es el marco sobre el cual es posible develar con amplitud parte de los complejos procesos desarrollados por la educación superior en Venezuela y todos los autores toman en referencia algún componente histórico en su trabajo. Los diversos artículos, además de nuestros análisis, son la base de esta introducción del número especial que la UDUAL me ha solicitado coordinar. Tal vez lo dominante sea esa mirada, tal vez nostálgica, además de comparativa, en los análisis de la situación actual que mira el presente y especialmente el futuro, de cara a lo que fue y ha dejado de ser y del curso que ha tomado la educación superior y el país y del cual todos tenemos una mirada particular desde sus impactos en nuestros países. Esta es también, sin duda, parte de nuestra lectura para comprender y analizar el presente de la educación superior en Venezuela y con ello también sus tendencias futuras.

\section{Marco general}

Ya antes, Rama (2015), hemos analizado que la educación superior en Venezuela se caracteriza por fases claramente delimitadas. En tal sentido afirmábamos que:

Tres etapas muy marcadas tiene la dinámica de la relación entre la educación superior privada y la educación superior en el país. Visto en el largo plazo Venezuela muestra una relación similar de crecimiento entre la matrícula total, la pública y la privada. Entre 1960 y 2008, en el largo periodo de 37 años, la media anual de incremento de la matrícula total de la educación superior fue de $9.65 \%$ anual, en tanto que la expansión de la educación pública ha sido de $9.21 \%$ anual y la privada de $11.63 \%$, lo cual implica una varianza muy baja. Sin embargo, si uno toma períodos, constata claramente tres etapas. Una primera marcada por una estabilidad de la cobertura privada entre 1960 , cuando es un $11.4 \%$ del total, y 1980, año en que alcanza a 12.9\%. Durante esos 10 años ambos sectores crecieron igual. Desde 1980 hasta 2002, encontramos una segunda fase marcada por un crecimiento sostenido mayor de la educación superior privada, cuya incidencia en el total pasa de $12.9 \%$ en 1980 al $43.9 \%$ en el año 2002, cuando alcanza su cenit. A partir de allí, se inicia la tercera fase en curso actual, caracterizada por un sector superior público en forma superior al sector privado. En esta fase, ya para 2008, la incidencia del sector privado había bajado al $27 \%$ del total.

Desde ese momento, se inició lentamente una nueva fase que se consolidó entre el año 2012 y el momento actual, que es el centro de nuestra reflexión, pero que se basa en las etapas anteriores referidas, no solo por la dinámica de la educación superior privada, sino porque, a su vez, esta se ajusta a la política de educación superior y a las características de la educación superior pública. 
Luis Fuenmayor fija el punto de inflexión en el año 2007, con el cambio de los equipos políticos y la irrupción de un nuevo paradigma que abandonó el equilibrio en la búsqueda entre calidad y equidad, y dota de una visión más político-ideológica a la política universitaria.

\section{La cobertura masiva}

La educación superior en Venezuela históricamente tuvo una alta tasa de cobertura. Una creciente dotación de recursos públicos, la alta diferenciación institucional con la creación de universidades experimentales en los setenta, una importante cobertura de estudios a distancia especialmente por el rol de la Universidad Nacional Abierta, un peso destacado del sector de estudios no universitarios, y un desarrollo significativo de la educación privada cuya génesis se localiza desde inicios de los años cincuenta con la creación de la Universidad Católica y desde los setenta fue dando paso a la aparición de universidades privadas, tanto de élites como de absorción de la demanda. Los años de la bonanza agregaron un potente sistema de becas nacionales e internacionales, tanto por parte de las universidades como de organismos públicos.

Sin embargo, los procesos de selección también alimentaron un bolsón de estudiantes que concluían el bachillerato y que no ingresaban a las universidades públicas ni privadas que para los años dos mil se contaron en cerca de medio millón, que alimentaron la irrupción de un nuevo sector para cubrir estas demandas insatisfechas a través de un modelo de acceso llamado Aldeas Universitarias y, posteriormente, Universidades Territoriales. Ello, junto a nuevas universidades como la Universidad Bolivariana y la transformación de la pequeña Universidad de las Fuerzas Armadas en una universidad de ingreso masivo ampliaron aún más la cobertura y permitieron a Venezuela ser el país con la mayor matrícula de la educación superior en la región.

El cambio de la cobertura hacia un tercer sector no solo se expresó en las nuevas plataformas institucionales creadas, sino que, además, a través de una política de centralización de los procesos de admisión y de un cupo de estudiantes asignados por el Ministerio de Educación se impulsó un cambio en la composición social de los estudiantes de las universidades tradicionales. Amalio Rafael Sarco Lira Barreto nos plantea la posibilidad de procesos arbitrarios sin indicadores de calidad por el carácter inauditable de dichos procesos.

El inicio del proceso, presumiblemente, implicó una transferencia de estudiantes al sector privado que buscaron calidad de los procesos, en tanto ello impactó sobre los criterios de calidad de las universidades tradicionales, y posteriormente con la crisis económica, esto podría haber impactado en el campo de la composición social y en procesos de emigración de la clase media estudiantil.

Para el año 2016, el 25.15\% de la población de más de 25 años tiene al menos estudios de nivel 6 según el Instituto de Estadística de la UNESCO, uno de los indicadores más elevados de América Latina y, al tiempo, constituye un elemento más complejo ante la crisis económica y la ausencia de oportunidades de empleo de profesionales y técnicos que caracteriza 
el escenario del desempleo en el país. Para el 2009, último año en el cual la UNESCO tiene información, la matrícula alcanzaba a 2,150 millones de estudiantes con una cobertura bruta de $79.53 \%$, la más alta de la región e, incluso, superior a la de Estados Unidos, en un contexto donde la media de la tasa de cobertura regional alcanzaba a 40\%. La educación superior venezolana era de casi el doble de la cobertura promedio a nivel regional. A partir de allí empezó su caída. Primero imperceptible al bajar a 79.15\% en el 2010 y luego, estrepitosa, más allá de que desde entonces se carece de datos oficiales.

\section{La diferenciación institucional fragmentada}

La diferenciación era un eje significativo de la educación superior del país, y se amplió desde la irrupción del cambio constitucional que impulsó el gobierno de Chávez y continuó Maduro; se crearon nuevas universidades nacionales (Bolivariana y de las Fuerzas Armadas) y múltiples universidades especializadas en diversos campos disciplinarios, así como se llevó a cabo la transformación de los Institutos Tecnológicos y Superior Universitarios prexistentes en Universidades Politécnicas Territoriales. Este proceso de diferenciación fue, más que un proceso técnico de cobertura a demandas y ofertas sectoriales, un proceso político que creó un tercer sector de la educación superior del país. Tulio Ramírez lo define acertadamente como un sector paralelo.

Además del sector autónomo de cinco universidades, y del sector privado, se creó este nuevo sector bajo control del gobierno, que incluso se expresó en una asociación de rectores diferenciada ARBOL (Asociación de Rectores Bolivarianos) diferenciada de la tradicional AVERU (Asociación Venezolana de Rectores de Universidades). Este sector se dotó de una importante plataforma física, tanto por la vía de la transferencia de los bienes del sector petrolero que en el 2002 se redujo de 40 mil a 20 empleados como resultado de la expulsión de las personas que participaron en la huelga petrolera de entonces, como por la vía de la sesión de cuarteles militares y la inversión en nuevos campi universitarios, especialmente en el interior del país. Esta diferenciación tuvo como eje la conformación de un sector público con fuerte control político, con menos niveles de calidad, más focalizado en sectores excluidos del acceso y más dependiente de la regulación pública y que tuvo un peso más destacado en la cobertura apoyado en planes de becas estudiantiles. Cónsono con ello, el propio acceso laboral al sector público comenzó a estar reservado a egresados de este nuevo sector con un perfil ideológico gubernamental.

Esta diferenciación institucional se realizó amparada al marco normativo de 1971 de universidades experimentales, cuya autonomía estaba limitada a un decreto habilitante del poder ejecutivo que nunca se produjo, e incentivado el acceso a través de un amplio sistema de becas con fuertes orientaciones políticas para los sectores de bajos ingresos.

La destacada participación del sector privado en todo el periodo se redujo a través de una política de no autorización de creación de nuevas ofertas, de regulación de los precios de las matrículas y del establecimiento de exigencias de funcionamiento y regulaciones salariales. De alcanzar cerca 
del $40 \%$ al $45 \%$ de la cobertura, el sector privado se redujo enormemente, y a pesar de la ausencia de datos estadísticos fiables, puede estar por debajo del $20 \%$ o $15 \%$ de la cobertura.

Al modelo tradicional dual de la educación superior de América Latina, la dinámica política agregó una fragmentación del sector público entre un sector autónomo y otro no autónomo, entre uno tradicional, en general de élites, frente a un sector público de alta absorción de demanda y sin regulación de calidad. El eje de una educación superior trinaria (pública, privada e internacional) fue aquí dividida entre un sector público autónomo, uno privado sin fines de lucro, y otro público estatal.

Como afirma Luis Bravo, estas Universidades Politécnicas Territoriales (UPT) que abarcan múltiples campos de estudio y abordan las necesidades de su contexto territorial conforman una nueva geopolítica nacional y su proceso de creación incluso tuvo apertura de universidades en la crisis en el 2018, y a la fecha, ha derivado en la creación de 26 upt en todo el territorio nacional.

\section{La creciente regulación política}

Tradicionalmente, el sistema de educación superior se auto regulaba a través de la Ley de Universidades que otorgaba un peso relativo a las diversas universidades y que tendía a generar una lógica corporativa. La creación de múltiples universidades cambió la hegemonía en el Consejo Nacional de Universidades y, al tiempo, se creó un Ministerio de Educación Superior. Más allá de los diversos nombres que ha tenido, este ministerio expresa la injerencia de la política gubernamental en la regulación que ha atravesado la dinámica de la gobernanza de la educación superior en la región.

En este caso, el paradigma ha sido el modelo de educación superior cubana, por el cual las universidades se han constituido en expresiones muy alineadas a la política oficial. El ministerio ha tenido crecientes roles y cometidos en torno a la regulación y fiscalización que han limitado la autonomía tradicional de la educación superior en materia de habilitar sus ofertas, el acceso, el egreso, y el funcionamiento en general, tanto académico como administrativo y, sin duda, el control del gasto.

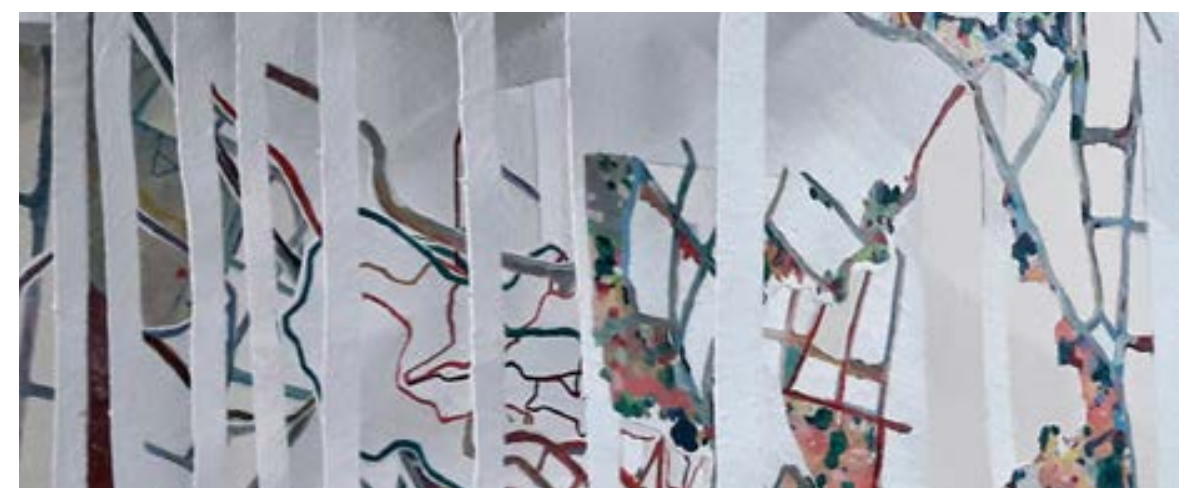


La Constitución de 1999 había establecido un marco por el cual las universidades no tradicionales podían acceder a la autonomía, pero ello no se produjo y, al contrario, desde entonces la autonomía se redujo lenta pero sistemáticamente, y se constituyeron en aparatos o brazos ejecutores de las políticas del Ministerio de Educación. La regulación ha sido, además, más compleja para su análisis por la falta de información pública para evaluar su coherencia con los problemas reales.

\section{El acotamiento de los instrumentos de mercado}

Las universidades venezolanas, como la mayor parte de las universidades de la región, han tenido una débil articulación con los mercados. Su relación dominante en el sector público ha sido a través de su oferta profesional y el cobro de las matrículas de posgrado y, en el sector privado, aun cuando también eran limitadas, eran más amplias por múltiples sistemas de acuerdos, tenían dinámicas de pertinencia más articuladas por la vía de los aranceles. Pero el eje dominante es una articulación por oferta y demanda de conocimientos asociados a la docencia.

Tradicionalmente, las universidades venezolanas se gestionaban a través de fundaciones o asociaciones de lucro. La regulación tuvo un enfoque limitante al rol del mercado en la asignación de recursos que se expresó en una de sus dimensiones contra la existencia del lucro, así como en la regulación de las tarifas, las transferencias y el gasto universitario, tanto sobre las universidades públicas como, especialmente, sobre las privadas. La política contra la mercantilización fue un mecanismo de control de las universidades que pasaron a tener dificultades de acceso a las divisas por su alta regulación y estatización, lo que derivó en una reducción significativa de la internacionalización de las universidades, de su participación en proyectos de investigación internacionales y de acceso a becas o actividades de movilidad externa. Venezuela se fue desligando de la comunidad académica regional e internacional y sus accesos pasaron a estar regulados por el Ministerio de Educación y mucho más acotados a una articulación con Cuba.

A medida que la dinámica económica se fue estatizando y que el sector privado tuvo limitaciones por expropiaciones, por topes de tarifas y de sus ofertas, el empleo profesional se localizó de manera dominante en el sector público. Se produjo una segmentación creciente del mercado de trabajo profesional en el cual el sector privado era el menor empleador y estaba limitado a las universidades autónomas y privadas; en tanto que el sector público se alimentó dominantemente de la oferta profesional del nuevo sector universitario. La polarización política se trasladó al mercado de trabajo, cuyas características comenzaron a alimentar una creciente emigración de profesionales y técnicos. La regulación de los salarios docentes también comenzó a impactar en el flujo hacia el extranjero de profesores, al igual que las limitaciones a las empresas privadas comenzaron a impulsar la emigración de técnicos y profesionales del país. El sector universitario, especialmente aquel con autonomía relativa y de carácter privado, comenzó a tener un impacto negativo en términos de estudiantes y docentes que se expresó 
en una cada vez mayor caída de la matrícula. Una parte de ella se trasladó, incluso, hacia el sector universitario estatal.

Altamente demandante de recursos públicos, el sector universitario accedió a ellos de forma importante en la fase de crecimiento de los precios petroleros, pero fue cada vez más controlado en la estructura de sus gastos. Ello se produjo al tiempo que hubo de hecho una creciente transferencia de nuevos recursos al emergente tercer sector universitario más articulado al gobierno, con menor calidad y costos. Sin embargo, pronto se comenzaron a plantear limitaciones a las aportaciones monetarias a medida que la economía nacional comenzó a entrar en importantes cuellos de botellas por exceso de regulación, expropiaciones y falta de acceso a divisas para importar en el sector privado, así como por limitaciones de los precios y las cantidades exportadas de petróleo, que se habían constituido en la casi única fuente de ingresos de divisas del país. La caída de las inversiones externas, del turismo y de otras exportaciones coadyuvaron a la crisis final del país, y también de la educación superior de Venezuela. Crisis que se ha disparado en el último quinquenio, que no solo agravó el cuadro general que se conformó en la primera década del siglo XXI, sino que agregó otras complejidades que golpearon negativamente en la educación superior de Venezuela.

\section{La calidad de la educación superior}

Los indicadores de calidad de la educación superior en la región, en general, son débiles e indirectos. Venezuela carece de un sistema de aseguramiento de la calidad consolidado y no participa en el El Programa para la Evaluación Internacional de los Alumnos (PISA, por sus siglas en inglés). La ausencia de datos torna muy complejo su análisis. Sin embargo, se puede plantear que la opción de política pública se focalizó en equidad y control. La masificación del sistema fue en detrimento de la calidad e impactó tanto al sistema como a las tradicionales universidades de élite y de investigación. La eficiencia como indicador de calidad también fue afectada y el egreso, según el último dato registrado de la unESCO, que alcanzó a 101,400 en el año 2004, mostró una eficiencia de titulación relativa similar a la región, a partir de entonces comenzó a caer con aumentos de los abandonos o de falta de titulación. Desde ahí la eficiencia habría tendido a caer sistemáticamente.

Para el 2019, según el ranking de Scimago, 66 universidades eran relevadas, de las cuales, la Universidad Central de Venezuela (ucv) ostentaba el mejor posicionamiento en la lista en el número 1593. Las tradicionales universidades públicas junto con la Universidad Simón Bolívar, también públicas, encabezaban los rankings. De la lista, 8 de las primeras 20 universidades son privadas y, en general, se localizan después de las públicas autónomas. Sin embargo, los posicionamientos han caído sistemáticamente desde que se tiene medición. 
Cuadro 1. Ranking web de universidades

\begin{tabular}{|c|c|c|c|c|c|c|}
\hline Ranking & $\begin{array}{l}\text { Ranking } \\
\text { mundial }\end{array}$ & Universidad & $\begin{array}{l}\text { Presencia } \\
\text { (Posición) }\end{array}$ & $\begin{array}{l}\text { Impacto } \\
\text { (Posición) }\end{array}$ & $\begin{array}{l}\text { Apertura } \\
\text { (Posición) }\end{array}$ & $\begin{array}{l}\text { Excelencia } \\
\text { (Posición) }\end{array}$ \\
\hline 1 & 1593 & Universidad Central de Venezuela & 882 & 2,307 & 1,423 & 2,006 \\
\hline 2 & 1641 & Universidad de los Andes Mérida & 742 & 1,586 & 1,328 & 2,525 \\
\hline 3 & 1855 & Universidad Simón Bolívar Venezuela & 1,411 & 2,656 & 1,083 & 2,540 \\
\hline 4 & 2886 & Universidad del Zulia & 1,621 & 5,440 & 2,092 & 3,252 \\
\hline 5 & 3014 & Universidad de Carabobo & 1,653 & 4,467 & 2,022 & 3,811 \\
\hline 6 & 3858 & Universidad de Oriente Venezuela & 4,341 & 8,423 & 3,037 & 3,941 \\
\hline 7 & 3747 & $\begin{array}{l}\text { Universidad Centroccidental Lisandro } \\
\text { Alvarado }\end{array}$ & 2,545 & 7,408 & 2,600 & 4,139 \\
\hline 8 & 4524 & $\begin{array}{l}\text { Universidad Nacional Experimental } \\
\text { de los Llanos Occidentales Ezequiel } \\
\text { Zamora }\end{array}$ & 4,304 & 8,030 & 6,557 & 4,348 \\
\hline 9 & 3838 & $\begin{array}{l}\text { Universidad Nacional Experimental } \\
\text { del Táchira }\end{array}$ & 3,915 & 3,382 & 5,487 & 4,695 \\
\hline 10 & 11408 & Fundación La Salle Ciencias Naturales & 14,414 & 15,898 & 8,602 & 5,040 \\
\hline 11 & 3987 & Universidad Católica Andrés Bello & 1,653 & 3,715 & 4,449 & 5,040 \\
\hline 12 & 11864 & Universidad Santa María Venezuela & 23,379 & 14,686 & 8,602 & 5,198 \\
\hline 13 & 9537 & $\begin{array}{l}\text { Universidad Nacional Experimental } \\
\text { de Guayana }\end{array}$ & 6,134 & 12,833 & 8,602 & 5,198 \\
\hline 14 & 6775 & $\begin{array}{l}\text { Instituto de Estudios Superiores de } \\
\text { Administración }\end{array}$ & 6,072 & 8,422 & 8,602 & 5,198 \\
\hline 15 & 12578 & Universidad Católica del Táchira & 21,189 & 14,707 & 8,602 & 5,362 \\
\hline 16 & 5734 & Universidad Metropolitana Caracas & 4,465 & 7,492 & 7,294 & 5,362 \\
\hline 17 & 24782 & $\begin{array}{l}\text { Instituto Universitario de Tecnología } \\
\text { Federico Rivero Palacio }\end{array}$ & 23,303 & 26,548 & 8,602 & 5,648 \\
\hline 18 & 10772 & Universidad Monte Ávila & 7,966 & 11,628 & 8,602 & 5,648 \\
\hline 19 & 5186 & Universidad Nacional Abierta & 4,635 & 4,451 & 6,889 & 5,648 \\
\hline 20 & 24286 & $\begin{array}{l}\text { Instituto Universitario de la Frontera } \\
\text { IUFRONT }\end{array}$ & 21,951 & 24,245 & 8,602 & 6,115 \\
\hline
\end{tabular}

Fuente: Consultado en octubre de 2019. https://www.webometrics.info/es/Latin_America/Venezuela?sor$\mathrm{t}=$ asc\&order=Excelencia\%20\%28Posici\%C3\%B3n $\% 2 \mathrm{~A} \% 29$

\section{La crisis final del modelo económico social y universitario}

La tragedia venezolana en el último quinquenio es una derivación de la conformación de un mapa institucional, político y económico de los dos quinquenios anteriores (Rama, 2018). La polarización como mecanismo de legitimación y de construcción de hegemonías que se había localizado también en el sector universitario, a través de la conformación del referido nuevo sector público y la limitación a los dos sectores preexistentes ca-

14 Universidades $\mid$ núm. 83, enero-marzo 2020| UDUAL $\mid$ DOI:https://doi.org/10.36888/udual.universidades.2020.83.72 Claudio Rama $\mid$ Mirada a la educación superior en Venezuela 
racterizan al modelo dual latinoamericano. La crisis económica acaecida y de la cual no puede salir la economía venezolana sin un cambio muy radical de sus políticas, derivó en una mayor intensidad de las políticas previas dada por una mayor regulación y estrangulamiento de los sectores autónomos y privados que redujeron su matrícula y expulsaron sus cuadros académicos. El achicamiento tajante del producto bruto interno vació la demanda de profesionales y técnicos, e impactó en sus salarios, lo que derivó en una emigración masiva; primero de capas medias y después de sectores de menos ingresos, especialmente de aquellos que no accedían a los planes sociales que estaban reservados a afinidades políticas, cada vez más precisos y focalizados a través de las Comunas Populares que siguieron el eje de los Comité de Defensa de la Revolución Cubana.

La crisis económica afectó, sin duda, en los recursos y la pérdida de docentes y estudiantes, pero también impactó en la inseguridad de los campi universitarios y el robo. La educación superior en Venezuela se ha caracterizado por un perfil urbanístico asociado a la existencia de campi universitarios, con consecuencias en el sector público en la capital y en el interior, como en el sector privado. Ellos comenzaron a ser objeto de robos y vandalismo permanente que implicaron el cuasi vaciamiento de sus equipos técnicos, así como de mucha de su infraestructura eléctrica, sanitaria y de gestión.

La pérdida de capacidades de docencia e investigación ha sido, al tiempo, la pérdida de capital humano por la altísima emigración que alcanzaría a cerca de 5 millones. Según ha expresado Claudia Vargas (2018):

[...] se estima que al menos 4 de 10 profesores universitarios han renunciado a la Universidad en Venezuela y más del $60 \%$ de ellos ha salido del país según la Asociación de Profesores UCV, lo cual significa la pérdida de su capital intelectual, el cierre de líneas de investigación y el deterioro en el mediano y largo plazo en el cumplimiento de la actividad docente. En el caso de los estudiantes, es cada vez mayor la cifra de deserción dentro de las carreras regulares y también el incremento en trámites asociados a legalización para procedimientos consulares y de apostilla de sus egresados. En ambos, perdemos capital humano, intelectual y fuerza de trabajo con la que no podrá contar Venezuela para su desarrollo en perspectiva.

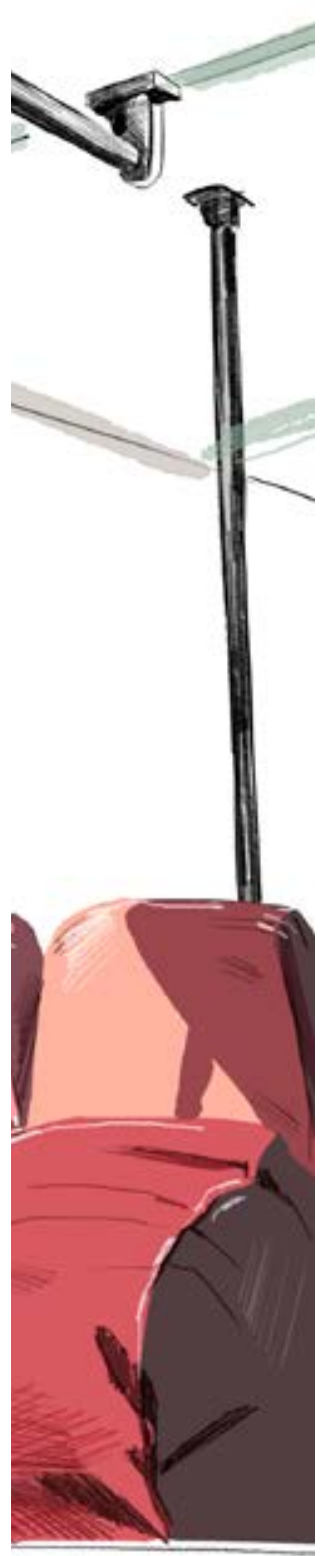

El alto nivel previo de dotación de capital humano en el cual la sociedad venezolana alcanzaba una media de estudios por persona al interior de la población económicamente activa, muestra el grado de pérdida de recursos humanos para el país, pacientemente construidos durante décadas de su plataforma institucional. Hay en tal sentido un cambio en los parámetros generales de la sociedad dados por menos demandas profesio- 
nales laborales, menos producto bruto y, por ende, menos producto per capita y mayores lógicas estatales.

Tal proceso derivó en crecientes manifestaciones estudiantiles durante el quinquenio y fuertes niveles de represión. Prácticamente las luchas estudiantiles han sido uno de los componentes que han acompañado el último quinquenio, y cada una de estas grandes etapas de revueltas ha sido aplacada con represión y muertos durante un tiempo. Este ciclo, a su vez, ha derivado en mayores impulsos emigratorios, especialmente de estudiantes.

\section{Los posibles caminos futuros}

La prospectiva necesariamente obliga a mirar a la educación superior sobre diversidad de escenarios sujetos a variables políticas. En uno de los escenarios de estos futuribles, la continuación del decrecimiento económico, la caída del empleo privado, las restricciones al empleo público y la inseguridad permiten visualizar un escenario de continuación y agravamiento de la crisis universitaria con impactos en el proceso de emigración. Los mercados laborales y, especialmente, los mercados profesionales se ajustan a través de procesos migratorios. Esta diáspora de venezolanos con un mayor perfil educativo, más allá de ser un fenómeno a escala global, tiene una mayor intensidad en América Latina, con diversos impactos, pero que crecientemente se aprecian políticas de limitación dada la dimensión y las complejidades de las reacciones en los diversos mercados laborales. El flujo migratorio es mayor ante las crecientes restricciones del gran mercado de absorción de demanda laboral que han sido tradicionalmente los Estados Unidos. El flujo venezolano está siendo incluso acompañado, y en algunos países, superado por el flujo cubano al anularse las políticas de "pies mojados, pies secos" que otorgaban residencias automáticas a los cubanos. La presión por la revalidación y homologación de estudios ha impactado en todos los sistemas universitarios en la región, acostumbrados a dimensiones muy inferiores de esos procesos a escala local.

En tal sentido, se podría considerar que la educación superior venezolana se está ajustando a un nuevo escenario de empleo y recursos que no ha dejado de impactar en la caída de los estudios en las universidades, especialmente en las privadas. La emigración junto a las políticas de admisión centralizadas también está impactando en el cambio social de las matrículas en el marco de estrategias políticas y se puede prever una continuación del cambio de la composición social del estudiantado.

\section{Referencias}

Rama, Claudio (2015). La nueva dinámica de la educación superior privada en Venezuela. Revista Pizarrón Latinoamericano, vol. 1, núm. 2, Caracas.

Rama, Claudio (2018). La destrucción de la universidad venezolana. Diario La República, Montevideo.https://www.republica.com.uy/la-destruccion-de-la-universidad-venezolana-id675005/

Vargas, Claudia (2018). La migración y su efecto sobre las universidades. especialment, Centro Gumilla. Caracas. http://revistasic.gumilla.org/2018/la-migracion-venezolana-y-sus-efectos-en-la-educacion-universitaria/ 